\title{
Localization of Dibromophenol in DPPC/Water Liposomes Studied by Anomalous Small-Angle X-ray Scattering
}

\author{
Zoltán Varga, ${ }^{\dagger}$ Attila Bóta, ${ }^{*} \dagger$ and Günter Goerigk ${ }^{\ddagger}$ \\ Institute of Physical Chemistry, Budapest University of Technology and Economics, \\ Müegyetem rkp 3, H-1521 Budapest, Hungary, and Institute of Solid State Physics, Research Centre Jülich, \\ D-52425 Jülich, Germany
}

Received: March 21, 2006; In Final Form: April 27, 2006

The localization of 2,4-dibromophenol molecules along the bilayer normal was investigated by anomalous small-angle X-ray scattering (ASAXS) with synchrotron radiation. The ASAXS measurements, executed at three different energies, provide the separation of the scattering of the bromine atoms of dibromophenol molecules from that of the whole system. Using a full $q$-range model, the localization of the dibromophenol molecules was characterized at a lower (0.1) and a higher (1) dibromophenol/lipid molar ratio corresponding to the gel and to the interdigitated phases of the vesicle matrix, respectively.

\section{Introduction}

In recent years, the effect of brominated phenol compounds on living organisms has become a field of interest. ${ }^{1-7}$ It is known that bromophenols are present in a wide range of different kinds of environment. For example, these chemicals occur in sponges and algae and also in other marine organisms, and they can be found in the blood of fishes and mammals, too.

The ecological role of these molecules is still not clear, but it has been shown that they have hormone-like effects and disrupt the cellular $\mathrm{Ca}^{2+}$ homeostasis in endocrine cells. ${ }^{6,7}$ Recently, the importance of the elucidation of the brominated phenols' effect has been increased not only by their natural occurrence in the organisms but also by their industrial application as fungicides and flame retardants (e.g., 2,4,6tribromophenol, tetrabromobisphenol A). These brominated contaminants act on basic cellular functions via complex interactions, which depend on many parameters; thus, it is worth examining the effect of bromophenols on separated components of the real cells. Consequently, vesicles (or liposomes) are frequently used as model membranes to study the effect of guest molecules on biological membranes.

In this paper, the localization of 2,4-dibromophenol in a multilamellar vesicle system constituted from 1,2-dipalmitoyl$s n$-glycero-3-phosphatidylcholine (DPPC) and water is studied at 0.1 and $1 \mathrm{DBP} / \mathrm{DPPC}$ molar ratios. It was previously reported that the accumulation of lipophilic compounds like dichlorophenols causes lateral heterogeneity and cluster formation in the double lipid layers. ${ }^{8,9}$ Presumably, the localizations of the dichloro- and dibromophenol molecules in the vesicle system are the same because of their similar chemical behavior. ${ }^{10} \mathrm{We}$ have studied the distribution of DBP molecules in vesicles with the anomalous small-angle X-ray scattering (ASAXS) method, which proved to be an excellent technique to determine the localization of atoms/molecules in a surrounding media. In other

* To whom correspondence may be addressed. Tel.: +3614632473 Fax: +36 1463 3767. E-mail: abota@mail.bme.hu.

Budapest University of Technology and Economics.

$\doteqdot$ Research Centre Jülich. words, by using ASAXS one can overcome the problem of separating the small-angle scattering of the guest molecules from that of the whole system. The basis of the method is the energy dependency of the scattering factors of the atoms, which are complex quantities and show a strong variation with the X-ray energy in the vicinity of the absorption edge of the atom under consideration, which is the bromine in our case.

\section{Experimental Section}

The method of anomalous small-angle X-ray scattering has been used in a great variety of fields, e.g., in the study of alloys, ${ }^{11}$ examining thin films of electrocatalysts, ${ }^{12}$ protein research, ${ }^{13,14}$ and describing the distribution of counterions around macroions and polyacrylate chains. ${ }^{15-17}$ In the latter paper, a method was introduced to separate the scattering of the guest atoms from that of the whole system. Hereinafter, this method will be reviewed with the specification for radially ordered systems.

From the small-angle X-ray scattering's point of view, a multilamellar vesicle can be considered as a stack of layers averaged over all orientations in the three-dimensional space; consequently, the scattered intensity can be expressed as

$$
I(q)=S(q)|F(q)|^{2} / q^{2}
$$

where $q$ denotes the magnitude of the scattering variable, $q=$ $4 \pi \sin \theta / \lambda ; F(q)$ is the bilayer form factor, and $S(q)$ is the structure factor of the layers. ${ }^{18,22,23} S(q)$ represents the contribution of the layer structure, while $F(q)$ is the Fourier transform of the bilayer electron density (ED) profile.

Generally, the form factor of a bilayer with additive guest atoms can be written as $F(q)=F^{\text {vesicle }}(q)+F^{\text {guest }}(q)$ where

$$
\begin{gathered}
F^{\text {vesicle }}(q)=\int_{-d / 2}^{d / 2} \Delta \rho^{\text {vesicle }}(z) \cdot \mathrm{e}^{-i q z} \mathrm{~d} z \\
F^{\text {guest }}(q)=\int_{-d / 2}^{d / 2} \Delta \rho^{\text {guest }}(z) \cdot \mathrm{e}^{-i q z} \mathrm{~d} z
\end{gathered}
$$

Here, $d$ is the bilayer repeating unit, and $\Delta \rho^{\text {vesicle }}(z)$ and 
$\Delta \rho^{\text {guest }}(z)$ are the average excess electron densities of the lipid layers and the guest molecules along the bilayer normal

$$
\begin{gathered}
\Delta \rho^{\text {vesicle }}(z)=\left(f_{\text {lipid }}-\rho^{\text {water }} V^{\text {lipid }}\right) u(z) \\
\Delta \rho^{\text {guest }}(z)=\left[\left(f_{0, \text { guest }}-\rho^{\text {water }} V^{\text {guest }}\right)+f_{\text {guest }}^{\prime}(E)+\right. \\
\text { if } \left.f_{\text {guest }}^{\prime \prime}(E)\right] v(z)
\end{gathered}
$$

Here, $\rho^{\text {water }}$ is the electron density of the solvent; $V^{\text {lipid }}$ and $V^{\text {guest }}$ are the volumes of the lipid layers and the guest molecules, while $u(z)$ and $v(z)$ are the radial average of the particle densities of lipids and guest molecules. For the sake of simplicity in the following, we will denote $f_{\text {guest }}^{\prime}$ and $f_{\text {guest }}^{\prime \prime}$ with $f^{\prime}$ and $f^{\prime \prime}$, respectively, since we only have to consider the anomalous dispersion corrections of the guest molecules, or more precisely of their resonant atoms. The molecular scattering factor of lipids, $f_{\text {lipid }}$, is nearly energy-independent, but that of the atoms of the guest molecules shows a strong variation with the energy in the vicinity of the absorption edge of the atom under consideration.

With the introduced quantities, one can rewrite the form factor of the system as a sum of a resonant, energy-dependent, and a nonresonant, energy-independent term: $F(q)=U(q)+\left(f^{\prime}(E)\right.$ $\left.+i f^{\prime \prime}(E)\right) V(q)$, where $V(q)=\int v(z) \exp (-i q z) \mathrm{d} z$ is the geometrical part of the form factor of the bromine atoms of the bromophenol molecules. Then, the measured intensity can be calculated as follows:

$$
\begin{aligned}
I(q, E)=\left\{U^{2}(q)+2 f^{\prime}(E) U(q) V(q)+\right. & {\left[f^{\prime 2}(E)+\right.} \\
& \left.\left.f^{\prime \prime 2}(E)\right] V^{2}(q)\right\} \frac{S(q)}{q^{2}}
\end{aligned}
$$

From this, the difference of two scattering curves, measured at two different energies, will be the following:

$$
\begin{aligned}
\Delta I\left(q, E_{1}, E_{2}\right)= & \left\{2\left[f^{\prime}\left(E_{1}\right)-f^{\prime}\left(E_{2}\right)\right] U(q) V(q)+\left[f^{\prime 2}\left(E_{1}\right)-\right.\right. \\
& \left.\left.f^{\prime 2}\left(E_{2}\right)+f^{\prime \prime 2}\left(E_{1}\right)-f^{\prime \prime 2}\left(E_{2}\right)\right] V^{2}(q)\right\} \frac{S(q)}{q^{2}}
\end{aligned}
$$

To eliminate the mixed term, a measurement at a third different energy should be performed, and the separated scattering of bromine atoms can be expressed as follows:

$$
\begin{gathered}
V^{2}(q) \frac{S(q)}{q^{2}}=\frac{1}{C\left(E_{1}, E_{2}, E_{3}\right)}\left[\frac{\Delta I\left(q, E_{1}, E_{2}\right)}{f^{\prime}\left(E_{1}\right)-f^{\prime}\left(E_{2}\right)}-\frac{\Delta I\left(q, E_{1}, E_{3}\right)}{f^{\prime}\left(E_{3}\right)-f^{\prime}\left(E_{3}\right)}\right] \\
C\left(E_{1}, E_{2}, E_{3}\right)=f^{\prime}\left(E_{2}\right)-f^{\prime}\left(E_{3}\right)+\frac{f^{\prime \prime 2}\left(E_{1}\right)-f^{\prime \prime 2}\left(E_{2}\right)}{f^{\prime}\left(E_{1}\right)-f^{\prime}\left(E_{2}\right)}- \\
\frac{f^{\prime \prime 2}\left(E_{1}\right)-f^{\prime \prime 2}\left(E_{3}\right)}{f^{\prime}\left(E_{1}\right)-f^{\prime}\left(E_{3}\right)}(6)
\end{gathered}
$$

According to eq 6, with ASAXS technique one can access the scattering of the guest atoms, so the distribution of these molecules in the vesicles can be directly observed.

Sample Preparation. Synthetic high-purity 1,2-dipalmitoylsn-glycero-3-phosphatidylcholine (DPPC) and 2,4-dibromophenol (DBP) were obtained from Avanti Polar Lipids (U.S.A.) and from Sigma (Germany), respectively. The pure DPPC without further purification was mixed with crystallized DBP in conformity with the different DBP/DPPC ratios, and then, deionized, triple quartz-distilled water was added to the system to gain a lipid concentration of $20 \mathrm{w} / \mathrm{w} \%$. The mixtures were

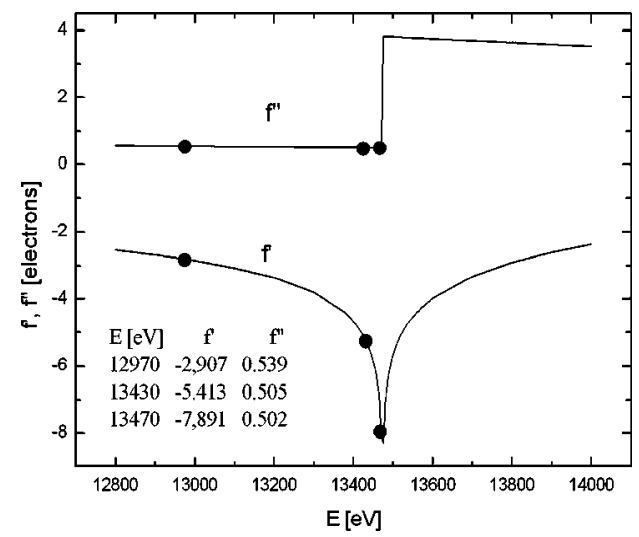

Figure 1. Anomalous dispersion corrections for bromine obtained by Cromer-Liberman calculations. ${ }^{20,21}$

kept at $45^{\circ} \mathrm{C}$ and vortexed intensively, then quenched to $4{ }^{\circ} \mathrm{C}$. This process was repeated 40 times to get a homogeneous liposome system. The X-ray measurements were performed using Plexiglas-walled (without significant small-angle scattering) sample holders; for precise incubation, thin water flow in front of the walls was used.

ASAXS Experiments. Anomalous small-angle X-ray scattering (ASAXS) measurements were carried out at the Jusifa (B1) beamline ${ }^{19}$ at HASYLAB (DESY, Hamburg) in the scattering variable $(q=4 \pi \sin \theta / \lambda)$ regime of $0.02-0.4 \AA^{-1}$. The measurements were made with a two-dimensional detector, at the energies 12970,13434 , and $13470 \mathrm{eV}$, close to the absorption edge of bromine at $13474 \mathrm{eV}$. These energies were chosen on the basis of an absorption measurement on DBP crystal. The net scattering data collected at different energies were computed to the same abscissa, normalized to the primary beam intensity, and corrected for transmission. Finally, the scattering curves have been calibrated to absolute units of macroscopic cross sections (e.u. $/ \mathrm{nm}^{3}$ ). For data evaluation, the anomalous dispersion corrections of $\mathrm{Br}$ were calculated according to Cromer and Liberman ${ }^{20,21}$ (Figure 1). The values of $f^{\prime}$ and $f^{\prime \prime}$ at the energies used are also shown in Figure 1.

\section{Results and Discussion}

By dispersing DPPC molecules in water, centrosymmetric multilamellar vesicles are formed spontaneously. According to the one-dimensional order (in radial direction), the scattering pattern of the system exhibits at least five Bragg peaks. In the case of the DPPC/water system doped with DBP molecules, depending on the concentration, the regular structure is damaged, but the periodicity is still present. As a result of the guest molecules, only two Bragg reflections can be observed on the scattering patterns. In Figure 2, scattering curves of the systems with 0.1 and $1 \mathrm{DBP} / \mathrm{DPPC}$ molar ratios are shown. All the measurements were carried out at $T=20^{\circ} \mathrm{C}$, which corresponds to the nonrippled gel phase $\left(\mathrm{L}_{\beta}{ }^{\prime}\right)$ of the pure DPPC/water system. The squares denote the total scattering of the system measured at $12970 \mathrm{eV}$, the farthest energy from the absorption edge of bromine (there is almost no anomalous effect from DBP molecules at this energy). Comparing the total scattering curves, one can identify drastic changes, which originate in the different phases of the systems induced by the different ratios of DBP molecules. At the lower concentration, DBP has a perturbation effect on the correlation of lamellae. The average periodicity (d) is $67.8 \AA$, which is larger than that of the regular nonrippled gel phase $(64.2 \AA)$. At higher concentration, the interdigitated form of the gel phase has appeared, as it can be recognized from the $d=50.7 \AA$ repeating distance. In that phase, the 

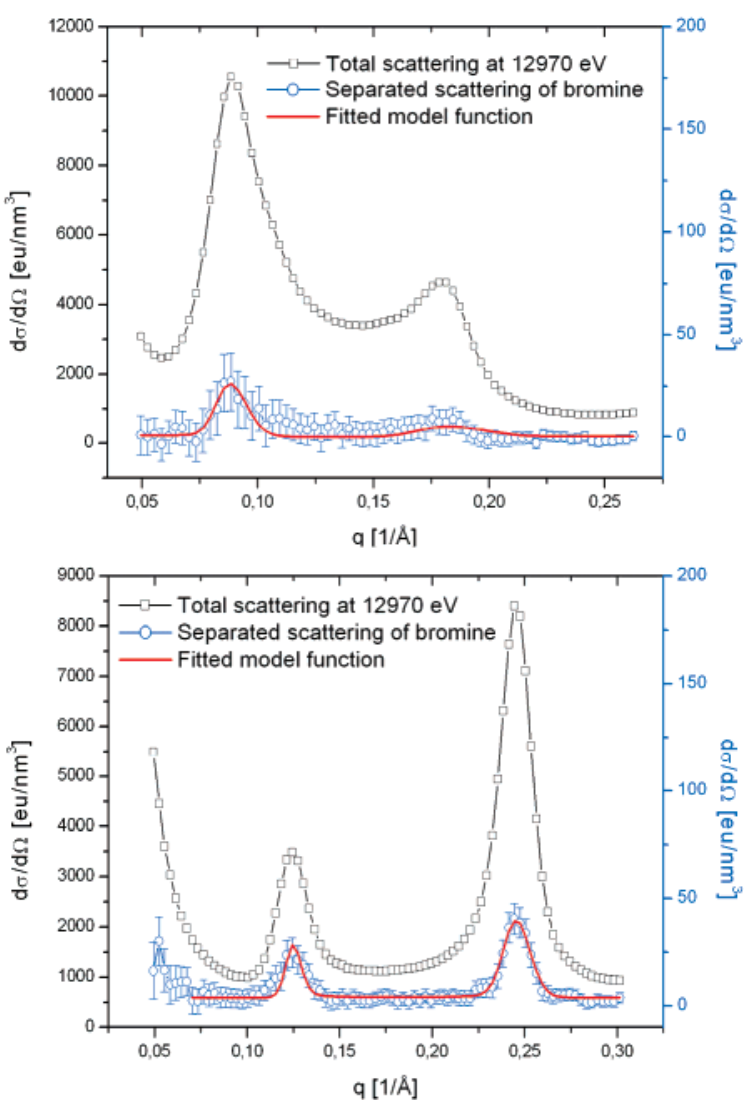

Figure 2. ASAXS measurements of DBP/DPPC systems at 0.1 (top) and 1 (bottom) molar ratios. Left ordinate belongs to the total scattering curves; right ordinate belongs to separated scattering curves from bromine atoms of DBP molecules.

hydrocarbon chains of the two lipid layers are fused, making it possible to form tighter packing. The ratios of the maximal intensities of the Bragg peaks are also different in the cases of the two DBP concentrations as a consequence of the changes in the radial electron densities of the bilayers (i.e., on one hand, the geometrical arrangement of the lipids and also the DBP molecules is changed, and on the other hand, drastic local enlargement in the electron density has been occurred by the DBP molecules).

The separated intensities of DBP molecules for the two DBP/ DPPC ratios, derived from measured data by using the righthand side of eq 6, are shown in Figure 2. Bragg reflections can be observed on these separated curves at the same position as on the total scattering curves, which provides clear evidence of the periodic displacement of DBP molecules corresponding to the periodicity of the vesicles.

To get more detailed information about the localization of DBP molecules in the bilayers, we performed a simple model fitting. ${ }^{22,23}$ For these calculations, the structure factor from paracrystalline theory was used, which takes the packing disorder into account

$$
S_{\mathrm{PT}}(q)=N+2 \sum_{k=1}^{N-1}(N-k) \cos (k q d) \exp \left(-k^{2} q^{2} \Delta^{2} / 2\right)
$$

where $N$ is the number of layers and $\Delta$ is the mean square fluctuation of the bilayers. The latter originates from the DebyeWaller temperature factor, but now, we consider it as the effect of the guest molecules.

The conventional way to determine the bilayer ED profile is to use Fourier synthesis on the Bragg intensities, which were previously corrected for $S(q)$ and the Lorentz factor $q^{2}$ according
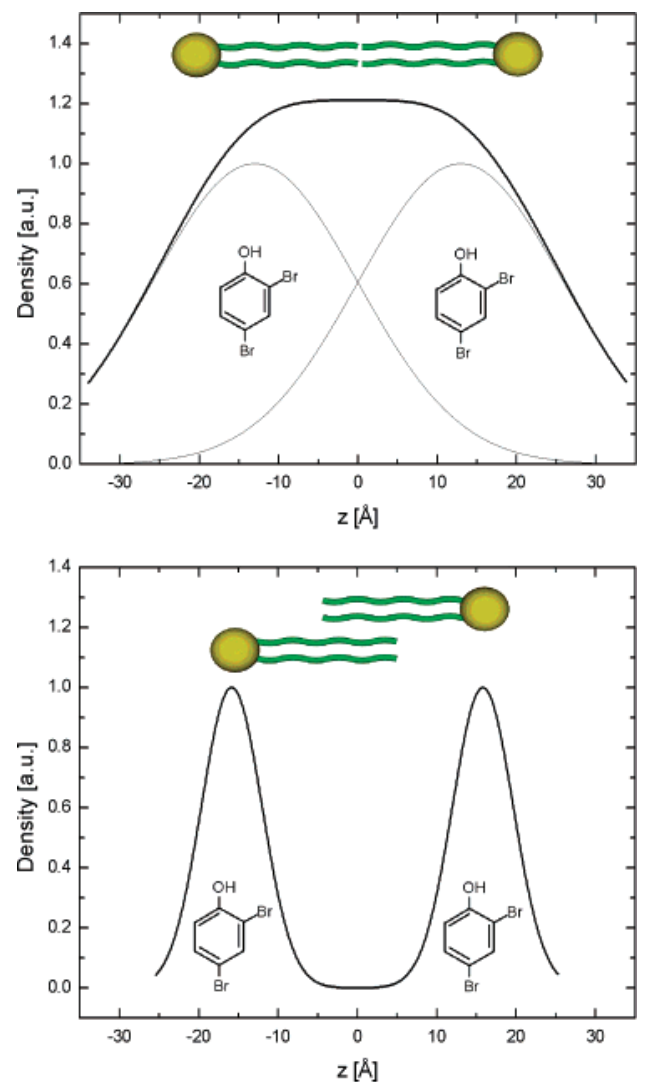

Figure 3. Particle densities along the bilayer normal at 0.1 (top) and 1 (bottom) DBP/DPPC molar ratio. The positions of the lipid molecules are also shown in the figure.

TABLE 1: Fitting Results: Number of Bilayers $N$, Repeating Unit $d$, Mean Square Fluctuation of the Bilayers $\Delta$, Width and Position (from the bilayer Center) of the Gaussian Function $\sigma$ and $z_{\mathrm{G}}$, Respectively

\begin{tabular}{cccccc}
\hline DBP/DPPC & $N$ & $d$ & $\Delta$ & $\sigma$ & $z_{\mathrm{G}}$ \\
\hline 0.1 & $25 \pm 1$ & $67.8(87)$ & $5.4(33)$ & $9.1(59)$ & $12.9(74)$ \\
1 & $19 \pm 1$ & $50.7(67)$ & $1.4(44)$ & $2.6(99)$ & $15.8(28)$
\end{tabular}

to eq 1 . This method, however, can be used only if the scattering pattern shows at least four diffraction orders. In our case, we have to consider a model ED profile because of the reduced number of Bragg peaks.

For modeling the ED profile along the bilayer normal, we have used a Gaussian model, which is based on describing the electron density as a sum of Gaussian functions. We have used only two Gaussian functions per bilayer located symmetrically to the center of the bilayer, because only the contribution of the guest molecules is present on the separated scattering curves. In the adumbrated case, the geometrical part of the form factor of the guest molecules will be the following:

$$
V(q)=\sqrt{2 \pi}\left[2 \sigma \exp \left(-\sigma^{2} q^{2} / 2\right) \cos \left(q z_{\mathrm{G}}\right)\right]
$$

where $\sigma$ and $z_{\mathrm{G}}$ are the width and the position from the bilayer center of the Gaussian function. The model fitting was performed using lsqcurvefit routine of MATLAB (The MathWorks, Inc.).

The fitted model functions agree convincingly with the measured data as can be seen in Figure 2 (The results of the fits are summarized in the Table 1). According to the form factor in eq 8 , the distributions of the guest molecules along the bilayer normal are shown in Figure 3.

These results clearly show that the DBP molecules are embedded in the lipid layers. In the case of the lower DBP/ 
DPPC ratio, the localization of the guest molecules is less determined than in the case of the higher ratio, where their positions are more localized to the vicinity of the headgroups.

\section{Conclusions}

The location of 2,4-dibromophenol molecules in DPPC/water liposomes was studied by the ASAXS technique at 0.1 and 1 DBP/DPPC molar ratios. The ASAXS curves measured at three different energies provided the derivation of the scattering contribution of the guest molecules. On the basis of a Gaussian model, these separated curves were interpreted, giving the possibility to determine the distributions of the bromophenols along the bilayer normal. In the case of the higher DBP concentration, the displacement of the guest molecules is characterized by a sharp distribution function pointing out the essential role of these molecules in the formation of the interdigitated phase.

This study reveals the utility of the ASAXS method in examining the distribution of a third component in a lamellar system, in the concrete case, in DPPC/water liposomes. However, this method can be applied in a great variety of these kinds of systems, such as diblock copolymers, which have high interest in nanoindustry.

Acknowledgment. This work was supported by the Hungarian Scientific Funds OTKA (T 43055) and by the Contract RII3CT-2004-506008 of the European Community at DESY/ HASYLAB.

\section{References and Notes}

(1) Kotterman, M.; van der Veen, I.; van Hesselingen, J.; Leonards, P.; Osinga, R.; de Boer J. Biomed. Eng. 2003, 20, 425.
(2) Lincoln, D. E.; Fielman, K. T.; Marinelli, R. L.; Woodin, S. A. Biochem. Syst. Ecol. 2005, 33, 559.

(3) Ríos, J. C.; Repetto, G.; Jos, A.; del Peso, A.; Salguero, M.; Cameán A.; Repetto, M. Toxicol. in Vitro 2003, 17, 635.

(4) Legler, J.; Brouwer, A. Environ. Int. 2003, 29, 879.

(5) Wollenberger, L.; Dinan, L.; Breitholtz, M. Environ. Toxicol. Chem. 2005, 24, 400 .

(6) Hassenklöver, T.; Predehl, S.; Pilli, J.; Ledwolorz, J.; Assmann, M.; Bickmeyer, U. Aquat. Toxicol. 2006, 76, 37.

(7) Olsen, C. M.; Meussen-Elholm, E. T. M.; Holme, J. A.; Hongslo, J. K. Toxicol. Lett. 2002, 129, 55.

(8) Sikkema, J.; de Bont, J. A.; Poolman, B. Microbiol. Rev. 1995, $59,201$.

(9) Csiszár, Á; Klumpp, E.; Bóta, A.; Szegedi, K. Chem. Phys. Lipids 2003, 126,155

(10) Bóta, A.; Szegedi, K.; Goerigk, G.; Csiszár, Á.; Haubold, H.-G.; Subklew, G. HASYLAB Annual Reports 2002.

(11) Goerigk, G.; Williamson, D. L. Solid State Commun. 1998, 108 , 419 .

(12) Vad, T.; Hajbolouri, F.; Haubold, H.-G.; Scherer, G. G.; Wokaun, A. J. Phys. Chem. B 2004, 108, 12442.

(13) Phillips, J. C.; Wlodawer, A.; Goodfellow, J. M.; Watenpaugh, K. D.; Sieker, L. C.; Jensen, L. H.; Hodgson, K. O. Acta Crystallogr. 1977, A33, 445 .

(14) Stuhrmann, H. B. Acta Crystallogr. 1980, A36, 996.

(15) de Robillard, Q.; Guo, X.; Dingenouts, N.; Ballauf, M. Macromol. Symp. 2001, 164, 81 .

(16) Guilleaume, B.; Ballauf, M.; Goerigk, G.; Witteman, M.; Rehahn, M. Colloid Polym. Sci. 2001, 279, 829.

(17) Goerigk, G.; Schweins, R.; Huber, K.; Ballauff, M. Europhys. Lett 2004, 66, 331

(18) Zhang, R.; Suter, R. M.; Nagle, J. F. Phys. Rev. E 1994, 50, 5047. (19) Haubold, H.-G.; Gruenhagen, K.; Wagener, M.; Jungbluth, H.; Heer, H.; Pfeil, A.; Rongen, H.; Brandenburg, G.; Moeller, R.; Matzerath, R.; Hiller, P.; Halling, H. Rev. Sci. Instrum. 1989, 60, 1943.

(20) Cromer, D. T.; Liberman, D. J. Chem. Phys. 1970, 53, 1891

(21) Cromer, D. T.; Liberman, D. Acta Crystallogr. 1981, A37, 267.

(22) Pabst, G.; Rappolt, M.; Amentisch H.; Laggner, P. Phys. Rev. E 2000, 62, 4000.

(23) Pabst, G.; Koschuch, R.; Pozo-Navas, B.; Rappolt, M.; Lohner, K.; Laggner, P. J. Appl. Crystallogr. 2003, 36, 1378. 